\title{
MUACLP: Modeling Unified Architecture of Cross Layer Parameters Strategy of Handoff for Next Generation Wireless Network
}

\author{
Hemavathi, S. Akhila
}

\begin{abstract}
The Quality of Service and Quality of experience is always a goal of future generation wireless systems. A suitably designed handoff plays an important role in achieving these goals. The traditional methods of handoff for wireless systems pose challenges in handling the smooth transition in the heterogeneous architecture of future generation architecture. This paper induces a model for synchronization of seamless handoff for a mobile user from a unified access point of a large cell to the small cell. The study is carried out considering a novel analytical model for facilitating a better form of vertical handoff mechanism in order to facilitate communication over $5 G$ networks. The modeling is carried out considering the efficiency of channel capacity as well as considering near real-time constraint of latency and throughput. The typical design assumption for the unified architecture of the model for the handoff is for future generation wireless network that includes various physical layer parameters. For an effective analysis, the study considers that the receiving node will be identified on the basis of the non-erroneous node as the idea is to increase the proper utilization of the network resources. The core agenda of the study will be to ensure that irrespective of any form of traffic intensity as well as variable resource availability, the proposed scheme should be able to offer an extensive communication with the seamless performance during handoff operation. The study outcome exhibits that the proposed system offers better vertical handoff performance in contrast to the existing system on various test cases.
\end{abstract}

Keywords : Vertical handoff, 5G communication, data delivery, constraint .

\section{INTRODUCTION}

The The continuous up-gradation and evolvement of wireless communication standards from $2 \mathrm{G}$ to $5 \mathrm{G}$ to provision seamless data service to be communicated through the Internet. The statistics of the research outcomes show that the user demand is continuously increasing for the data services by using various kinds of data-driven applications by mobile

Revised Manuscript Received on June 30, 2020.

* Correspondence Author

Hemavathi*, Electronics and Communication Engineering B.M.S College of Engineering, VTU, Bangalore, India. Email: hvathi.draj@gmail.com

S. Akhila, Electronics and Communication Engineering B.M.S College of Engineering, VTU, Bangalore, India.

(c) The Authors. Published by Blue Eyes Intelligence Engineering and Sciences Publication (BEIESP). This is an open access article under the CC BY-NC-ND license (http://creativecommons.org/licenses/by-nc-nd/4.0/) node users. The innovations and inventions in the global connectivity of various physical objects as the Internet of things (IoT) require communication with a high level of scalability and fault tolerance capacity. The fixed base station context in the traditional previous generation cellular network poses design constraints on the future generation networks due to the inflexible design of the equipment, whereas in the next-generation networks, the system must adaptive [1]. Therefore, the future generation wireless networks (FGWN) designs aim for the highly reliable with optimal quality of services (QoS) and Quality of Experience (QoE) balance to be synchronous with different date rates operating at varied frequency bands to make it highly scalable to offer large user base connectivity. So that the FGWN can provide spectrum with the large nodes and varied application support, the seamless handoff between the two distinguished cell architecture of past and future generation access points need to be designed efficiently by considering all the prominent parameters at the different layers of the communication. The final problem is connected with the management of the mobility factor. Basically, mobility management is used to explore the correct connectivity between mobile nodes with changing addresses. Unfortunately, this is a really challenging task as such problems need to be solved considering attributes from different layers apart from the data link layer [2]. One of the easiest mechanisms to look for the supportive wireless network during the vertical handoff process is to retain the interfaces in an active state. At the same time, all the inactive interfaces should always be avoided as there is larger resource consumption if the interface is always on active mode; eventually, there is no data packet to be received. Therefore, there is an essential need to evolve up with a more customized and user-centric mechanism that facilitates accessing diversified services as well as network irrespective of using single or multiple interfaces of radio [3]. The 5G communication system possess a device that is capable of operating different forms of the accessing system using diversified cards, facilitating the switching mechanism that is required between different interfaces. A system is required that can undertake decisions with higher intelligence for autonomous selection of interfaces in an active mode that suits well for a specific session of an application. However, the conditions of the network is another critical factor to affect the decision-making process in vertical handoff for facilitating an appropriate switching system from any ongoing session in active mode to any new active interface.

\section{Published By:}

Blue Eyes Intelligence Engineering \& Sciences Publication 


\section{MUACLP: Modeling Unified Architecture of Cross Layer Parameters Strategy of Handoff for Next Generation Wireless Network}

At present, there is various work being carried out towards vertical handoff management [4]. However, they are less focused on adopting the next generation of the communication system. Therefore, the proposed system aims to implement a system that considers all the necessary traits required to support the vertical handoff mechanism in the $5 \mathrm{G}$ networking system.

According to the proposed system, the vertical handoff is considered to be taken from macro-cells to small cells under the $5 \mathrm{G}$ networking system. The proposed system also adheres to the near real-time constraints of the $5 \mathrm{G}$ network that makes the implementation outcome more practical with more scope of applicability. The organization of the paper is as follows: Section II discusses about the existing literatures on different techniques for vertical handoff mechanism followed by a discussion of the proposed solution and the algorithm implementation in Section III and IV. Section V discusses result analysis. Finally, the conclusive remarks are provided in Section VI.

\section{THE BACKGROUND}

There have been various works that have been carried out towards the vertical handoff mechanism discussed in our prior work [5]. The recent work carried out $\mathrm{Bi}$ et al. [6] have performed the classification of signal strength information and various other mobility parameters for constructing the optimal network for effective support of handoff. Fachtali et al. [7] have used swarm intelligence for improving the decision in vertical handoff in the wireless system of communication. Study towards 5G communication system and handoff operation is carried out by Qiang et al. [8] using a multi-objective optimization method for facilitating network selection in the vertical handoff. The study carried out by Mahardhika et al. [9], and Li et al. [10] have carried out the vertical handoff operation considering multiple metrics. The work of Wang et al. [11] has presented a unique scheme of a selection of decision tree considering distributed vehicular communication for assisting in better handoff mechanism. The work of Chen et al. [12] have used q-learning method for offering better blocking performance over heterogeneous wireless networks to improve the quality of experience. A study toward heterogeneous wireless networks was also carried out by Yang et al. [13], where the authors have discussed a unique bandit model that uses the elimination of the state algorithm. Masek et al. [14] have considered the case study of 5G, where the sharing options for the spectrum are emphasized for better handoff operation in order to support the dynamic operation. Xue et al. [15] have presented a model-driven framework over multipath propagation in wifi-system. The work of Hseuh and Liu [16] has investigated the relationship between vertical and horizontal events of handoff considering cellular networks where the importance was given to investigate the cost of handoff operation. A study on handoff schemes is carried out by Kumar et al. [17], where the cognitive network is considered for performing spectrum handoff emphasizing over decision making for a better quality of services. Kustiawan et al. [18] have used fuzzy logic for formulating an effective decision for vertical handoff to offer better efficiency of operational time. Ma et al. [19] have constructed a decision tree using the linearized modeling method for enhancing the decision making process. Cheelu et al. [20] have also used fuzzy logic for exploring the better form of network configuration, supporting vertical handoff using a predictive method. The work carried out by Lin et al. [21] has used optimization techniques in order to address mobility problems in the next-generation network. Therefore, the existing system has been targeted to address various problems associated with the vertical network with diversified techniques where each method has its own benefits and own limitation. The next section outlines the research problems. By Bisio and Sciarrone [22] have presented a selection mechanism of an explicit network to carry out a vertical handoff mechanism. Another recent study of Coqueiro et al. [23] has used fuzzy logic for implementing an energy-efficient handoff mechanism in a wireless network for addressing quality of experience. Investigation towards vertical handoff over the ultra-dense network is carried out by Jia and Zheng [24], focusing on the optimal allocation of the resources with better control over traffic performance. Foukas et al. [25] have presented a technique where vertical handoff operation is carried out over shorter communication ranges with a special focus on the energy efficiency of the mobile device.

From literature survey the identified issues associated with the existing methods are as follows:

- The existing scheme of vertical handoff is restricted to mainly use fuzzy logic and common wireless networks in order to support the existing networking system.

- The conventional scheme has very less emphasis over the upcoming networking standards as well as mobile networking e.g., 5G with respect to vertical handoff.

- Research towards prioritizing communication over the high data rate of transmission is less studied over current times.

- Identification of an optimal decision-making scheme for vertical handoff without using the conventional iterative scheme is still an open-end problem.

Therefore, the proposed system aims at "Developing a cost-effective vertical handoff mechanism for facilitating better decision-making process without compromising the data forwarding performance in upcoming dynamic high transmission rate network. "'

\section{PROPOSED SOLUTION}

This part of the research work is an extension of our prior modeling [26]. The current work emphasizes the implementation of the vertical handoff in a network of high transmission rate i.e., 5G standards. The complete work is carried out when the mobile user from the macro-cells move towards small-cells in 5G networks. The adopted scheme of research is shown in Fig.1 


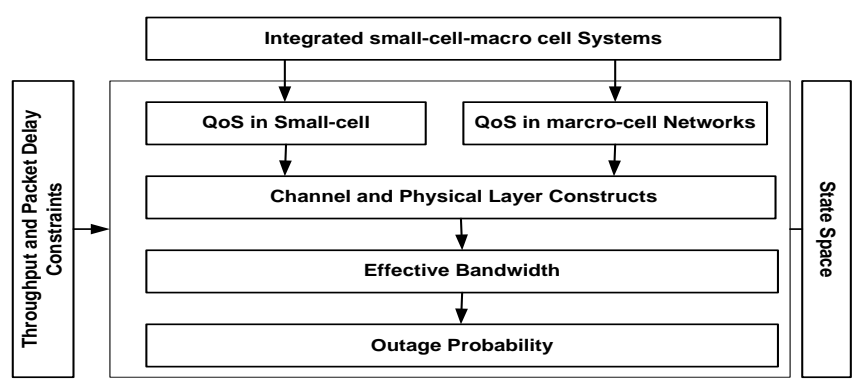

Fig. 1.Adopted Research Scheme

The study is carried out in the presence of near real-time constraints associated with the data delivery performance as well as latency in order to adhere to the applicability of the proposed system with the $5 \mathrm{G}$ networks. Therefore, the study considers an effective channel capacity that is optimally required to carry out a vertical handoff mechanism over the macro-cells in order to investigate the usage of the degree of utilization of the channel resources considering physical layers. For an effective analysis, the study considers that the receiving node will be identified on the basis of the non-erronous node as the idea is to increase the proper utilization of the network resources.

The proposed mechanism introduces a novel decision-making block that can permit certain network systems to have access to another form of the network on the basis of traffic and session time. The study also formulates different resource availability and allocation in macro-cell and small-cell. The study also introduces an effective channel capacity scheme in order to ensure uniform utilization of the resources. The study also uses both network and physical layer and their associated parameters in order to perform the investigation of the proposed study of vertical handoff. The core agenda of the study will be to ensure that irrespective of any form of traffic intensity as well as variable resource availability, the proposed scheme should be able to offer an extensive communication with the seamless performance during handoff operation. The next section outlines the system design and implementation.

\section{SYSTEM IMPLEMENTATION}

The typical design assumption for the unified architecture of the model for the handoff is for future generation wireless network that includes various physical layer parameters as listed in the table 1 . as time requires for each distinguish headers.

Table- I: Physical layer parameters and time taken

\begin{tabular}{|l|l|l|l|}
\hline Sl. No & Parameters & Values & Unit \\
\hline 1 & PHY-Header & $40-60$ & $\mu \mathrm{s}$ \\
\hline 2 & MAC-Header & $20-30$ & $\mu \mathrm{s}$ \\
\hline 3 & RTS & $10-20$ & $\mu \mathrm{s}$ \\
\hline 4 & CTS & $5-15$ & $\mu \mathrm{s}$ \\
\hline 5 & ACK & $5-15$ & $\mu \mathrm{s}$ \\
\hline
\end{tabular}

In the previous wireless network generation, the mechanism for the handoff takes the criteria and constraints of the cell only, whereas the FGWN operates at multi-layers that affect adversely due to uneven power distribution and heterogeneity of the cells architectures and their access points, as a result of this there exits computational overheads (complexity) as well degrades the quality of experience. The optimal usage of layer-wise resources ensures the balanced usage of the constraint spectrum that is a very critical requirement for channel resource utilization. The study deals with the problem associated with the design and development of a novel optimal handoff algorithm considering the performance metrics of speed, quality-of-services, and network preferences. The study considers the constraints of the utilization of channel resources.

\section{A. Small Cell with Access point for Wireless Network}

The cell region of the future generation wireless network $(F G W N)$, takes a random position of ' $\delta x$ ' and ' $\delta y$ ' with the communication range ' $R$ ' to generate a small cell positioning to meet the future generation $5 \mathrm{G}$ communication standard in order to comply its goal of reliability along with the desired data rate and reliability, thereby meeting the objective of extending the magnified side of the bandwidth signal so that the coverage can be extended to the wide ranges of the users or downlinks. The equation (1) and (2) is used to construct the localization construct of the small cell. Another predictor variable ' $\theta$ ' as a series with a small incremental value such that $\mathbf{0} \leq \boldsymbol{\theta} \leq \mathbf{2} \pi$

$$
\begin{array}{r}
\Delta \mathrm{x}=\mathrm{R} \cdot \operatorname{Cos}(\boldsymbol{\theta})+\boldsymbol{\delta} \mathbf{x} \\
\Delta \mathrm{y}=\mathrm{R} \cdot \operatorname{Sin}(\boldsymbol{\theta})+\boldsymbol{\delta} \mathbf{y}
\end{array}
$$

2D-Small cell $\leftarrow$ fcell $\left(\Delta \mathrm{x}, \Delta \mathrm{y},{ }^{\prime} \mathbf{R}\right.$ ')

The initial visualization of the small cell appears as shown in the figure 2. This cell structure is applicable to the any network including wireless local area network (WLAN), 4G as well as $5 \mathrm{G}$ based wireless network systems.

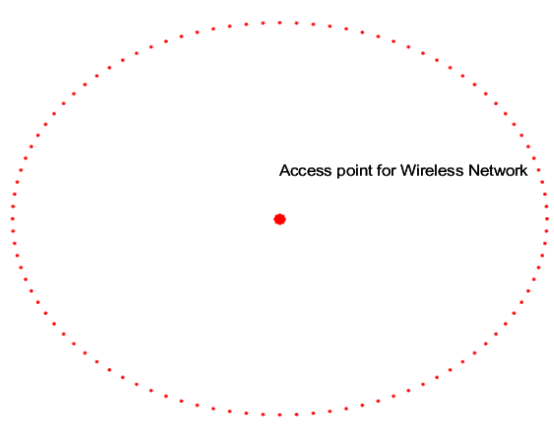

\section{Fig. 2.Small cell structure of future generation wireless network}

The FGWN will have backward and forward synchronization for the access technology including CDMA in $2 \mathrm{G}$ and $3 \mathrm{G}$, whereas $4 \mathrm{G}$ uses OFDMA(orthogonal frequency division multiple access) and SC-FDMA(single carrier frequency division multiple access) for downlink and uplink respectively, whereas $5 \mathrm{G}$ will evolve with the new access technology, to do mathematical modeling of the system a unified representation of the access points is represented by ' $\boldsymbol{\Phi}$ ', the architectural representation of the system model of a cell with the unified access point is shown in figure 3 .
Published By: Blue Eyes Intelligence Engineering \& Sciences Publication (C) Copvriaht: All riahts reserved. 


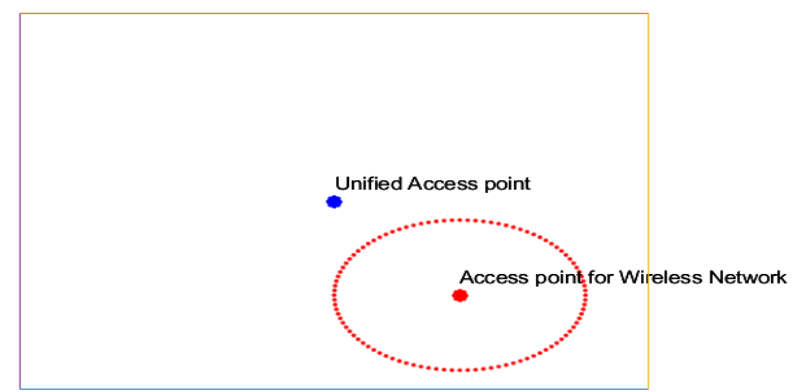

Fig. 3. Architectural representation of the system model of cell with unified access point

\section{B. Mobility model of the User}

The model uniquely designs a mobility model based on the probabilistic method of the mobility pattern of the cell or user device, where the independent variable for the mobile cell user $\left(\mathrm{U}_{\mathrm{m}}\right)$ starting location sets $\mathrm{S}_{\mathrm{L}}=\left\{\mathrm{X}_{l}, \mathrm{X}_{h}, \mathrm{Y}_{l}, \mathrm{Y}_{h}\right\}$ initializes its value ranging into the unified access point range $\left(R_{U A P}\right)$. The localization of the $U_{m}$ a dependent variable that depends upon the $\mathrm{S}_{\mathrm{L}}$ and to have a uniform probability of distribution that uses a uniformly distributed random generator function, where the $\mathrm{x}$ and $\mathrm{y}$ coordinates vector computations of $\mathrm{X}(\mathrm{Um})$ and $\mathrm{Y}(\mathrm{Um})$ uses the equ (4), and equ (5) respectively.

$$
\begin{gathered}
X\left(U_{m}\right)=\sum\left(X_{l}+\left(X_{h}-X_{l}\right)=f_{\text {rand }}(S) \ldots\right. \\
Y\left(U_{m}\right)=\sum\left(Y_{1}+\left(Y_{h}-Y_{D}\right)=f_{\text {rand }}(S)\right.
\end{gathered}
$$

The ' $\mathrm{S}$ ' is a seed in the uniform random generator function $f_{\text {rand }} 0$. The $U_{m}$ source localization is source. $U_{m} \leftarrow$ $X\left(U_{m}\right) \| Y\left(U_{m}\right)$, The mobile cell user $\left(\mathrm{U}_{\mathrm{m}}\right)$ visualization is shown in the figure 4.

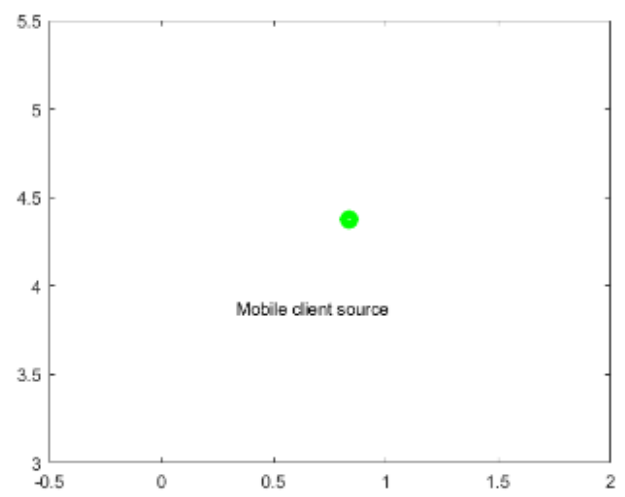

Fig. 4.Localization of the source of mobile cell user (Um) in the Mobility model

The destination of mobile cell user $\left(U_{m}\right)$ localization process for $\mathrm{D}_{\mathrm{x}} \cdot \mathrm{U}_{\mathrm{m}}$ and $\mathrm{D}_{\mathrm{y}} \cdot \mathrm{U}_{\mathrm{m}}$ is constructed with the variants of $\{\delta x, \delta y, R, \Delta x, \Delta y\}$, which is computed with the eq. (7) and eq. (8) respectively.

$$
\begin{array}{r}
D_{x}-U_{m}=\Sigma\left(\left[\delta_{x}-(R-1 / 2)\right]+\left[\delta_{x}-\left(\delta_{x}(R-1 / 2)\right)\right] * f(S)\right) \\
D_{y} \cdot U_{\mathrm{m}}=\Sigma\left(\left[\delta_{y}-(R-1 / 2)\right]+\left[\delta_{y}-\left(\delta_{y}(R-1 / 2)\right)\right] * f(S)\right)
\end{array}
$$

The random seed of the function as ' $\mathrm{S}$ ' generates a random value in both the cases of $U_{m}$ source and location points for validating the model as Monte Carlo simulation for the functionality test. The $U_{\mathrm{m}}$ destination localization is destination $\mathrm{U}_{\mathrm{m}} \leftarrow D_{x}-U_{m} \| D_{y}-U_{m}$, The mobile cell user $\left(\mathrm{U}_{\mathrm{m}}\right)$ source visualization along with the destination visualization is shown in the figure 5 . The final system model architecture is shown in figure 6 .

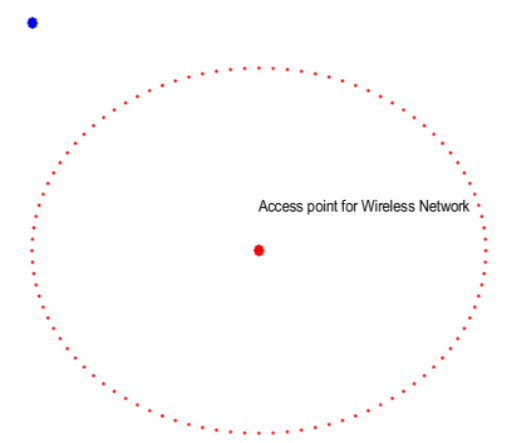

Fig. 5.Localization of the destination of mobile cell user (Um) in the Mobility model

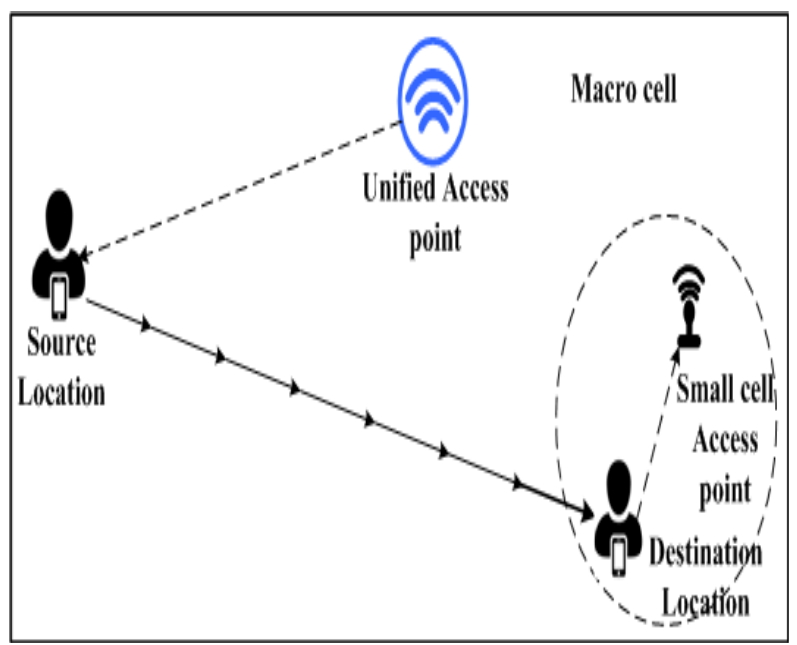

Fig. 6. Final architectural representation of the system model

The step computation, uses $\vec{S}$ and $\vec{D}$

$$
\begin{aligned}
& S P_{x} \leftarrow \vec{S}(\hat{i}) ; i=1 \\
& D P_{x} \leftarrow \vec{D}(j) s j=1 \\
& S P_{y} \leftarrow \vec{S}(k) ; k=2 \\
& D P_{y} \leftarrow \vec{D}(D) ; l=2 \\
& X_{\text {gtep }} \leftarrow f_{\text {ls }}\left(S P_{x^{x}} D P_{x^{3}} W\right) \\
& Y_{\text {step }} \leftarrow f_{l s}\left(S P_{y,} D P_{x,} W\right)
\end{aligned}
$$

The mobility pattern is mapped with the incremental movement as a random walk from the source to the destination point in such a way that the speed is controlled with a variable parameter. Therefore, with this deployment of the macro and the small cell access points along with the random walk model of mobility, the unified architecture is prepared to be synchronous for the FGWNs.

Published By:

Blue Eyes Intelligence Engineering

\& Sciences Publication

DOI: 10.35940/ijeat.E1091.069520

Journal Website: www.ijeat.org

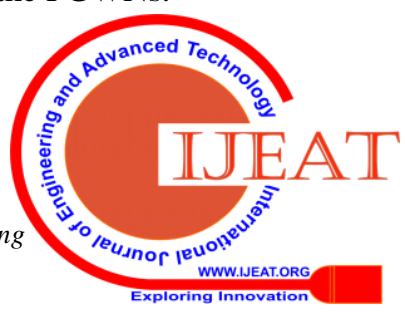


In order to validate the architecture, three processes, namely 1) Only Handoff, 2) only admission control, and 3) jointly admission control along with Handoff, is simulated. Section $\mathrm{V}$ describes the significance of its performance.

\section{UNIFIED ARCHITECTURE OF FGWN VALIDATION AND RESULTS}

The unified architectural model of FGWN is mathematically modelled and simulated on an efficient numecial computing platform, MATLAB, where different attributes of the physical layer, network layer frame specification, and unified access points specification are taken as an independent varibale. The simulation parametrs are listed in the table 1 and table 2 .

Table- II: Parameter for simulation

\begin{tabular}{|l|l|l|}
\hline Sl. No & Parameters & Values \\
\hline 1 & Acess Categories & $1,2,3,4$ \\
\hline 2 & Trasmission Opportunity & 5 \\
\hline 3 & $\begin{array}{l}\text { Arbitration interframe } \\
\text { space number }\end{array}$ & 1 \\
\hline 4 & $\begin{array}{l}\text { Minimum contention } \\
\text { window }\end{array}$ & 1 \\
\hline 5 & $\begin{array}{l}\text { Maximum contention } \\
\text { window }\end{array}$ & 32 \\
\hline 6 & Maximum backoff stage & $5,6,4,7,8,2,1,5,6,4$ \\
\hline 7 & $\begin{array}{l}\text { Constant probability of } \\
\text { collision }\end{array}$ & $\begin{array}{l}12,15,4,10,12,23,64, \\
87,4,21\end{array}$ \\
\hline
\end{tabular}

In 802.11 channel accesses, there are many mechanisms used to minimize the likelihood of frame collisions by multiple stations attempting to access the transmission medium simultaneously [27]. The HCF is an optional access method that may be used in addition to DCF, which was introduced to support QoS. HCF assimilated elements of both DCF and PCF mechanisms, creating a contention-based HCF method, called EDCA (Enhanced Distributed Channel Access method), and a contention-free HCF method, called HCCA. EDCA is a means of prioritizing contention-based wireless medium (WM) access by classifying 802.11 traffic types as 4 different Access Categories (AC). Whereas HCCA was designed to provide parameter-based contention-free transmission opportunities (TXOPs). The TXOP is used in wireless networks supporting the IEEE 802.11e Quality of Service enhancement and specifies the amount of time a station can send frames when it has won contention for the wireless medium. Used in both EDCA and HCCA modes of operation, the TXOP increases throughput and reduces the delay of QoS data frames via eliminating contention periods between transmissions.

The access categories have different channel access parameters, such as AIFS (Arbitration Interframe Spacing), duration, contention window size, and TXOP limit.There are 8 priority levels per station mapped to 4 access categories (AC) namely Background category, Best Effort category, video category and voice category each with different TXOP. Background and Best Effort categories must contend for the wireless medium for each and every frame they send and video category and voice category can send as many frames it can within the allotted time. Once its TXOP is done, it must contend for the wireless medium again if it has additional frames to send. Once that frame is sent, it must contend for the wireless medium again with the CCA (Clear Channel
Assessment), NAV(Network Allocation Vector), AIFS(Arbitration InterFrame space), and CW.

The purpose of an IFS (Inter frame space) is both to provide a time between frames to avoid interference as well as to add control and to prioritize frame transmissions [27].After a mobile station has observed an idle wireless medium with carrier sense (CS) for the appropriate IFS interval an additional deferral period must be observed before transmission of an data frames. The deferral period is known as a random backoff period and is selected at random by the mobile station from a window of possible values called a contention window (CW). In 802.11 CSMA/CA most important mechanism used to prevent collisions is the random backoff process.

The analysis is carried out with respect to three different schemes namely i) handoff without any admission control; ii) admission control without any vertical handoff scheme iii) joint handoff with admission control. In the legacy wireless network the contention characteristics imposes various challenges for achieving efficient radio access. The collision avoidance in MAC protocols with the backoff mechanism is tested with the optimal adjustment of the contention window to minimize the colloision probability in the proposed architecture of unified FGWN. The behavioural analysis of the backoff stages with the varying windows size is shown in the figure7 as below.

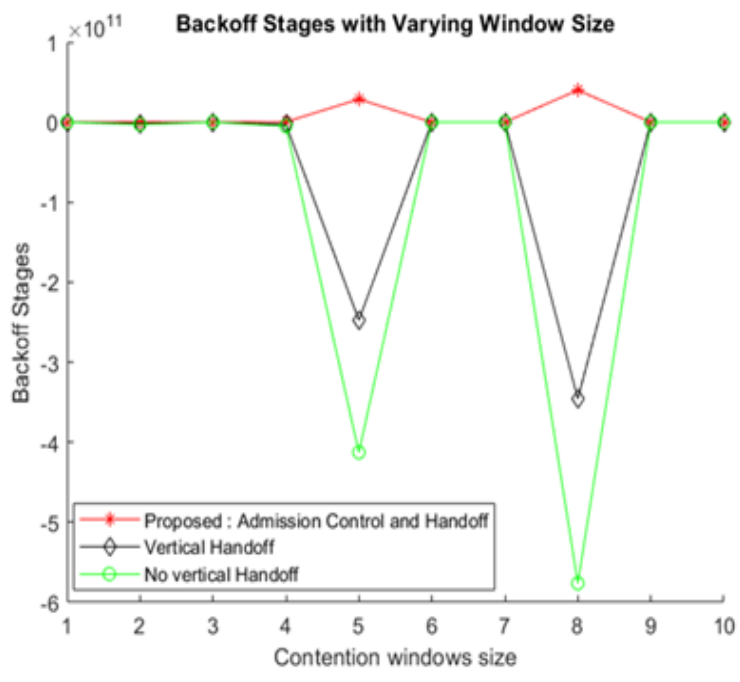

Fig. 6.Analysis of Backoff Counter

Figure 7 depicts the analysis of Backoff stages with varying contention window size. It can be observed from the graph that when the backoff stage is at zero, nodes start transmitting without any collision. As the number of nodes increases eventually, then there is more probability of collision in the existing system indicated by negative values in the above graph. In contrast to that proposed system has positive values which mean less collision probability, which validates the consistancy of the proposed method. The figure 8 shows the behaviour of the both existing methods along with the proposed method, the percentage probability of the packet transmission. The analysis of transmission probability graph with respect to the changing contention window range reveals that,

\section{Published By:}

Blue Eyes Intelligence Engineering \& Sciences Publication (C) Copvriaht: All riahts reserved. 


\section{MUACLP: Modeling Unified Architecture of Cross Layer Parameters Strategy of Handoff for Next Generation Wireless Network}

the proposed system attains higher probability as compared to the both existing systems. In the existing system, the scheme with the vertical handoff exhibits better probality of packet transmission as compare to the only admission control without handoff method.

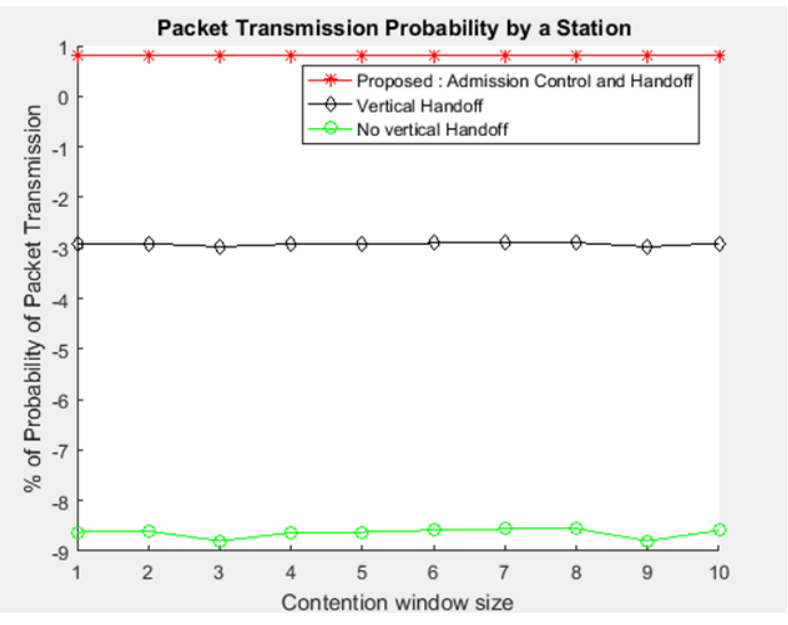

Fig. 7.Analysis of Transmission Probability offers better channel capacity as it exhibits higher backoff stage ( 0 to $\mathrm{m}$ ), where $\mathrm{m}>0$, so that the percentage of the packet transmission probability is higher in comparsion to the only admission control and only handoff. The outcome shows that if the proportion of traffic in a small cell is higher then it will increase the utilization of the proposed system. The prime reason is that proposed implementation can enhance the process of permitting certain incoming network in the small cells. The outcome clearly shows that the proposed system offers better outcomes in contrast to the existing system. The prime reason behind this outcome is that owing to the inclusion of the constraint associated with the data delivery in the proposed system, the future goal is to imporve the quality of the signal that should higher in contrast to the presence of interference over the receiving nodes which cannot be addressed in existing schemes. On the other hand, the proposed system offers optimal usage of the channel capacity, which results in maximized utilization of network resources, as seen in Fig.8. Moreover, channel capacity is a part of the quality of service parameters, which ensures that users of small-cell have a low mobility rate while that of macro-cell. Apart from this, the proposed system doesn't get much affected owing to the inclusion of different mobility patterns among the mobile nodes stating more sustenance towards dynamicity. Therefore, a stabilized receiver node can be expected when vertical handoff is being carried out from macro-cell to small-cell, and hence a cost-effective vertical handoff mechanism is presented in the proposed system.

\section{CONCLUSION}

In the future generation networks, there will be both macro and small cells having various kinds of access technologies. According to the proposed system, the vertical handoff is considered to be taken from macro-cells to small cells under the $5 \mathrm{G}$ networking system. The proposed study offers a significant level of quality of service performance in the
The study outcomes clearly show that the implementation

presence of signal outages over $5 \mathrm{G}$ networks. This proposed study contributes to utilizing the characteristics of the small and macro cells in order to offer a vertical handoff mechanism where an uninterrupted communication is retained over the $5 \mathrm{G}$ network. The main contribution of the proposed system are i) it jointly uses small cells and macrocells along with effective utilization of channel resources, ii) it considers the near real-time constraints associated with latency and throughput factor associated with transmission of heavier files over 5G network, iii) the outcome shows that proposed optimal scheme offers significant success over the access management during handoff whereas in existing system it is carried out by each network

\section{REFERENCES}

1. Rghioui, Amine, and Abdelmajid Oumnad. "Challenges and opportunities of internet of things in healthcare." International Journal of Electrical and Computer Engineering 8, no. 5 (2018): 2753.

2. Dong, Hu Jia, Raed Abdulla, Sathish Kumar Selvaperumal, Shankar Duraikannan, Ravi Lakshmanan, and Maythem K. Abbas. "Interactive on smart classroom system using beacon technology." International Journal of Electrical \& Computer Engineering (2088-8708) 9 (2019).

3. Brik, Vladimir, Arunesh Mishra, and Suman Banerjee. "Eliminating handoff latencies in 802.11 WLANs using multiple radios: Applications, experience, and evaluation." In Proceedings of the 5th ACM SIGCOMM conference on Internet Measurement, pp. 27-27. USENIX Association, 2005.

4. Neeraja, S., and A. Abhishiktha. "A comparative study on handover probability analysis for future HetNets." International Journal of Electrical \& Computer Engineering (2088-8708) 9 (2019).

5. Hemavathi, S. Akhila, "An Insight towards Trends and Effectiveness of Vertical Handoff Mechanism”, Research Gate, 2016

6. Bi, Yuming, Lei Tian, Mengmeng Liu, Zhenzi Liu, and Wei Chen. "Research on Joint Handoff Algorithm in Vehicles Networks." Chinese Journal of Engineering 2016 (2016).

7. El Fachtali, Imad, Rachid Saadane, and Mohammed ElKoutbi. "Vertical handover decision algorithm using ants' colonies for 4G heterogeneous wireless networks." Journal of Computer Networks and Communications 2016 (2016): 4.

8. Qiang, Li, Jie Li, and Corinne Touati. "A user centered multi-objective handoff scheme for hybrid 5G environments." IEEE Transactions on Emerging Topics in Computing 5, no. 3 (2016): 380-390.

9. Mahardhika, Gita, Mahamod Ismail, and Rosdiadee Nordin. "Vertical handover decision algorithm using multicriteria metrics in heterogeneous wireless network." Journal of Computer Networks and Communications 2015 (2015).

10. Li, Limin, Lin Ma, Yubin Xu, and Yunhai Fu. "Motion adaptive vertical handoff in cellular/WLAN heterogeneous wireless network." The Scientific World Journal 2014 (2014).

11. Wang, Shangguang, Cunqun Fan, Ching-Hsien Hsu, Qibo Sun, and Fangchun Yang. "A vertical handoff method via self-selection decision tree for internet of vehicles." IEEE Systems Journal 10, no. 3 (2014): 1183-1192.

12. Chen, Jiamei, Yao Wang, Yufeng Li, and Ershen Wang. "QoE-aware intelligent vertical handoff scheme over heterogeneous wireless access networks." IEEE Access 6 (2018): 38285-38293.

13. Yang, Bingtao, Xue Wang, and Zhihong Qian. "A Multi-Armed Bandit Model-Based Vertical Handoff Algorithm for Heterogeneous Wireless Networks." IEEE Communications Letters 22, no. 10 (2018): 2116-2119.

14. Masek, Pavel, Evgeny Mokrov, Krystof Zeman, Aleksey Ponomarenko-Timofeev, Alexander Pyattaev, Sergey Nesterov, Sergey Andreev, Jiri Hosek, Konstantin Samouylov, and Yevgeni Koucheryavy. "A practical perspective on 5g-ready highly dynamic spectrum management with lsa." Wireless Communications and Mobile Computing 2018 (2018).

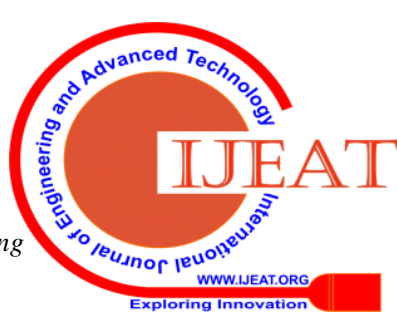


15. Xue, Chaojing, Wenzhong Li, Lingfan Yu, Jiacheng Shang, Xu Chen, and Sanglu Lu. "SERO: A Model-Driven Seamless Roaming Framework for Wireless Mesh Network With Multipath TCP." IEEE Transactions on Communications 67, no. 2 (2018): 1284-1296.

16. Hsueh, Shin-Ying, and Kuang-Hao Liu. "An equivalent analysis for handoff probability in heterogeneous cellular networks." IEEE Communications Letters 21, no. 6 (2017): 1405-1408.

17. Kumar, Krishan, Arun Prakash, and Rajeev Tripathi. "A spectrum handoff scheme for optimal network selection in nemo based cognitive radio vehicular networks." Wireless Communications and Mobile Computing 2017 (2017).

18. Kustiawan, Iwan, Chun-Yi Liu, and D. Frank Hsu. "Vertical handoff decision using fuzzification and combinatorial fusion." IEEE Communications Letters 21, no. 9 (2017): 2089-2092.

19. Ma, Bin, Dong Wang, Shuangguo Cheng, and Xianzhong Xie. "Modeling and analysis for vertical handoff based on the decision tree in a heterogeneous vehicle network." IEEE Access 5 (2017): 8812-8824.

20. Cheelu, D., Madda, R.B., Chen, C.Y., Krishna, P.V. and Yenduri, S., 2017. Intelligent vertical handoff decision strategy based on network performance prediction and consumer surplus value for next generation wireless networks. Iet Networks, 6(4), pp.69-74.

21. Lin, Po-Chiang, Lionel F. Gonzalez Casanova, and Bakary KS Fatty. "Data-driven handover optimization in next generation mobile communication networks." Mobile Information Systems 2016 (2016).

22. Bisio, Igor, and Andrea Sciarrone. "Fast Multiattribute Network Selection Technique for Vertical Handover in Heterogeneous Emergency Communication Systems." Wireless Communications and Mobile Computing 2019 (2019).

23. Coqueiro, Thiago, José Jailton, Tássio Carvalho, and Renato Francês. "A Fuzzy Logic System for Vertical Handover and Maximizing Battery Lifetime in Heterogeneous Wireless Multimedia Networks." Wireless Communications and Mobile Computing 2019 (2019).

24. Jia, Fan, and Xiaolin Zheng. "A Request-Based Handover Strategy Using NDN for 5G." Wireless Communications and Mobile Computing 2018 (2018)

25. Foukas, Xenofon, Kimon Kontovasilis, and Mahesh K. Marina. "Short-Range Cooperation of Mobile Devices for Energy-Efficient Vertical Handovers." Wireless Communications and Mobile Computing 2018 (2018)

26. Hemavathi S. Akhila, "FAVH: A Novel Technique of Faster Authentication in Vertical Handoff in WLAN and CDMA Network", International Journal of Scientific \& Engineering Research Volume 8, Issue 7, July-2017

27. Marcus Burton, "802.11 Arbitration -White Paper", CWNP (Certified Wireless Network Professional) September 2009 ,Version 1.00.

\section{AUTHORS PROFILE}

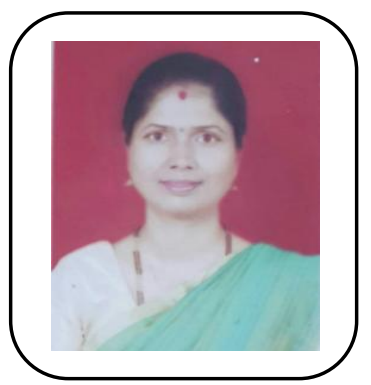

Hemavathi is working as an Assistant Professor in the department of Electronics and Communication Engineering at $\mathrm{B} \mathrm{M} \mathrm{S}$ College of Engineering, Bengaluru. She has completed her B.E in Electronics and Communication from Adi Chunchanagiri Institute of Technology, Chikmagalur, Kuvempu University, M.Tech from Acharya Institute of Technology, Bengaluru, VTU (Visvesvaraya Technological University). Currently she is pursuing Ph.D in the department of Electronics and Communication Engineering, B M S College of Engineering at VTU. She is a life member of Indian Society for Technical education (ISTE) since 2018. She has published papers in 5 International Journals, 1 International Conference and 4 National conferences.

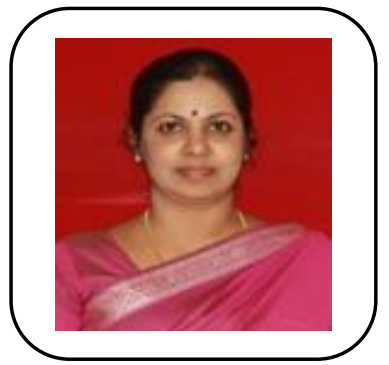

Dr. S. Akhila received her Bachelors in Electronics in the year 1988 and her Masters in Electronics in the year 1994 from University Visvesvaraya College of Engineering, Bangalore, India. She has completed her Ph.D. in the year 2013 from the Visvesvaraya Technological University (VTU) in Wireless Communication. Since 1995, she has been with B M S College of Engineering, where she is working as a Professor in the Electronics and
Communication Engineering Department. She is a life member of Indian Society for Technical education (ISTE). She has published papers in more than 4 National conferences, 6 International Conference and 25 International Journals. 